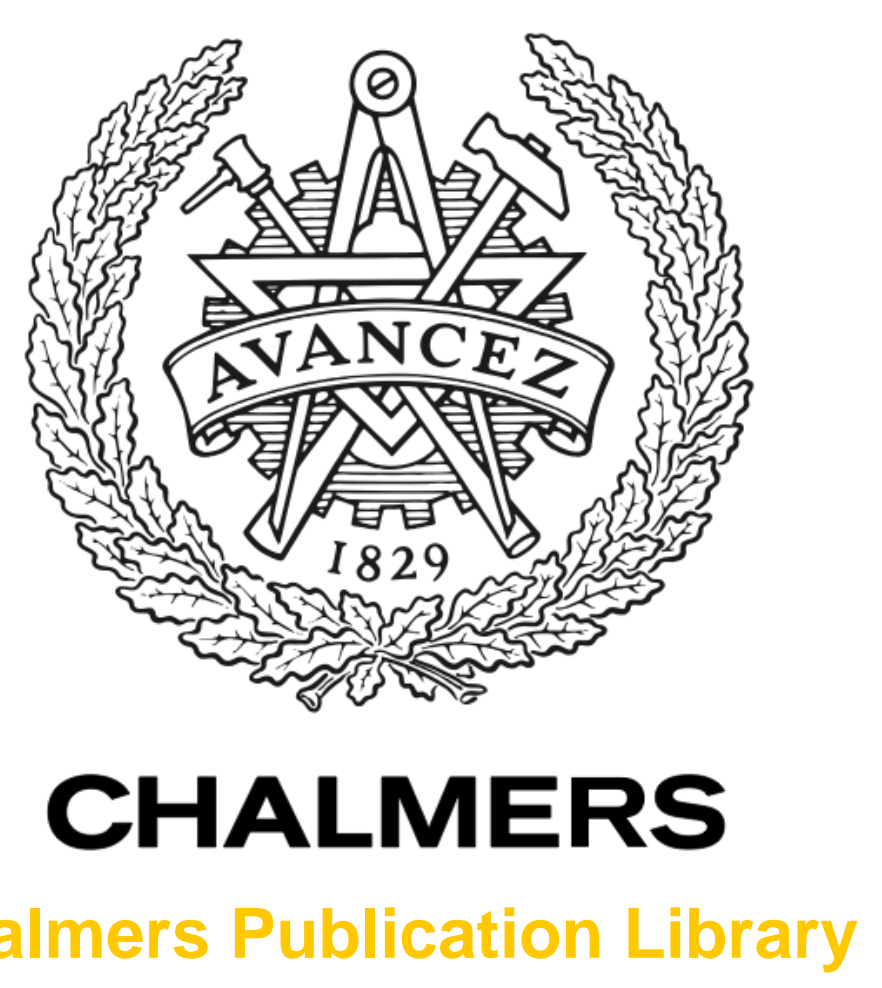

Chalmers Publication Library

\title{
Cancellation of drift kinetic effects between thermal and energetic particles on the resistive wall mode stabilization
}

This document has been downloaded from Chalmers Publication Library (CPL). It is the author's version of a work that was accepted for publication in:

Nuclear Fusion (ISSN: 0029-5515)

Citation for the published paper:

Guo, S. ; Liu, Y. ; Xu, X. et al. (2016) "Cancellation of drift kinetic effects between thermal and energetic particles on the resistive wall mode stabilization". Nuclear Fusion, vol. 56(7),

http://dx.doi.org/10.1088/0029-5515/56/7/076006

Downloaded from: http://publications.lib.chalmers.se/publication/240396

Notice: Changes introduced as a result of publishing processes such as copy-editing and formatting may not be reflected in this document. For a definitive version of this work, please refer to the published source. Please note that access to the published version might require a subscription. 


\title{
Cancellation of drift kinetic effects between thermal and energetic particles on the resistive wall mode stabilization
}

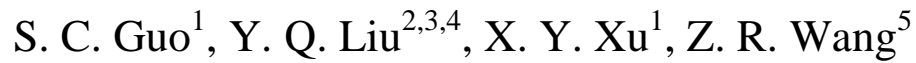 \\ ${ }^{1}$ Consorzio RFX Corso Stati Unit 4 - 35127 Padova, (Italy) \\ ${ }^{2}$ CCFE Culham Science Centre, Oxon OX14 3DB, UK \\ ${ }^{3}$ Southwestern Institute of Physics, PO Box 432, Chengdu 610041, China \\ ${ }^{4}$ Department of Earth and Space Science, Chalmers University of Technology, SE-412 96 Gothenburg, \\ Sweden \\ ${ }^{5}$ Princeton Plasma Physics Laboratory, Princeton, NJ 08543, USA \\ Corresponding author: yueqiang.liu@ccfe.ac.uk
}

\begin{abstract}
Drift kinetic stabilization of the resistive wall mode (RWM) is computationally investigated using MHD-kinetic hybrid code MARS-K following the non-perturbative approach [Liu et al., Phys. Plasmas 15, 112503 (2008)], for both reversed field pinch (RFP) and tokamak plasmas. Toroidal precessional drift resonance effects from trapped energetic ions (EIs) and various kinetic resonances between the mode and the guiding center drift motions of thermal particles are included into the self-consistent toroidal computations. The results show cancellation effects of the drift kinetic damping on the RWM between the thermal particles and EIs contributions, in both RFP and tokamak plasmas, even though each species alone can provide damping and stabilize RWM instability by respective kinetic resonances. The degree of cancellation generally depends on the EIs equilibrium distribution, the particle birth energy, as well as the toroidal flow speed of the plasma.
\end{abstract}

\section{Introduction}


It is well accepted that the resistive wall mode (RWM) instability is one of the main obstacles for achieving high beta, high bootstrap fraction plasmas in the so called advanced tokamak scenarios [1-3]. It is of crucial importance to understand the physics associated with the mode, as well as the potential ways to actively control the stability of the mode [4-6], for the purpose of not only expanding the operational space of plasma discharges, but also, perhaps even more critically for future fusion devices, avoiding major disruptions due to the onset of low- $n$, global RWMs. In the reversed field pinch (RFP) plasmas, the RWM instability, which is experimentally observed to grow exponentially [7], is often the limiting factor that terminates the discharge [8].

In this modelling work, we are mainly interested in the kinetic damping of the RWM, due to the mode resonances with drift motions of plasma particles - both thermal and energetic particles. Drift kinetic damping of the mode in tokamaks has been extensively studied during recent years [9-14], less so in RFPs [15-17]. However, most of the work has so far been focusing either on the thermal particle kinetic effects [10-19], or on the energetic particle (EP) effects alone [20-23]. A limited number of work considered the combined kinetic effects of both thermal and energetic particles on the mode [24], though essentially following the so called perturbative approach, in which the growth rate and real frequency of the mode are determined from an extended energy principle [9], but the eigenfunction of the mode is taken from computations based on the fluid theory. Few study is carried out to investigate the RWM stability following the non-perturbative approach, in the presence of both thermal and energetic particle kinetic resonances. The non-perturbative approach assumes that the eigenfunction of the RWM can be self-consistently modified by the drift kinetic resonances. This can only be realized either via full particle kinetic simulations, or via a self-consistent solution of both MHD and (drift) kinetic equations in a hybrid manner [10,13]. The latter has been adopted in the MARS-K code [10,22], which we use in this study.

We shall be primarily interested in the kinetic damping of the RWM in RFP plasmas, by both thermal and energetic particles. Most of the kinetic physics study for the RWM in RFPs has so far been focusing on thermal particles [15-17]. This is partly due to the fact that the existing RFP devices, such as RFX-mod and T2R, where the RWM instability can occur, do not have auxiliary heating systems that produce energetic 
ions (EIs). It is, however, plausible that future RFP devices will be equipped with auxiliary heating in order to achieve high temperature plasmas. In fact a significant amount of EIs are already generated in MST during the neutral beam injection (NBI) [25]. Neutral beams normally yield EIs with slowing down energy distribution but anisotropic particle pitch angle distribution. This aspect of anisotropy will also be addressed in the present work.

For the RWM, which is normally well phase locked to the surrounding resistive wall of the tokamak or RFP devices, the drift kinetic resonances occur essentially in the presence of toroidal plasma flow, which Doppler shifts the mode frequency. Thus the mode rotates in the plasma frame. In RFP plasmas, toroidal flow has been found to be able to stabilize the RWM according to the fluid theory, but at a speed of the Alfven velocity range [26]. Drift kinetic computations, with thermal particles alone, show that a flow at sound speed range is needed for the RWM stabilization in RFP plasmas [15]. Adding EIs contribution does not qualitatively alter this flow range, but quantitatively does, as will be shown in this work. Since the kinetic precession drift motion of trapped EIs has specific toroidal direction, it is important to consider the kinetic effects with both co- and counter-current flows. This issue is also addressed in the present study.

The main result from this investigation is the discovery of a cancellation effect, for the drift kinetic damping of the RWM, between the thermal and energetic particle contributions. This is observed in the non-perturbative MHD-kinetic hybrid computations for the RFP plasmas. Similar effect is also found for tokamak configurations, which we also report here for the purpose of comparison with the RFP results. It is plausible that similar cancellation effect can also be partially observed following the perturbative MHD-kinetic hybrid approach. In this work, however, we shall only follow the non-perturbative approach, which we believe to be more accurate in describing the drift kinetic effects on the RWM.

The next Section briefly describes the computational models adopted in this work. Section 3 reports the MARS-K modelling results for the $n=6$ RWM stability in a toroidal RFP plasma ( $n$ is the toroidal mode number), followed by Section 4 reporting the tokamak results for the $n=1$ RWM. Section 5 draws conclusion. 


\section{Models and formulations}

\subsection{Toroidal self-consistent MHD-kinetic hybrid model}

The MARS-K code numerically solves the linearized, single fluid MHD equations with self-consistent inclusion of drift kinetic resonances in toroidal geometry [10,22]. For a given curvilinear flux coordinate system $(s, \chi, \phi)$, and assuming that all the perturbations have the form $A(s, \chi, \phi, t)=A(s, \chi) \mathrm{e}^{-\mathrm{i} \omega t-\mathrm{i} n \phi}$, the MHD equations are written in the Eulerian frame

$$
\begin{aligned}
& -i(\omega-n \Omega) \xi=\mathbf{v}+(\xi \cdot \nabla \Omega) R^{2} \nabla \phi \\
& -i \rho(\omega-n \Omega) \mathbf{v}=-\nabla \cdot \mathbf{p}+\nabla \times \mathbf{Q} \times \mathbf{B}+\nabla \times \mathbf{B} \times \mathbf{Q}-\rho\left[2 \Omega \hat{\mathbf{Z}} \times \mathbf{v}+(\mathbf{v} \cdot \nabla \Omega) R^{2} \nabla \phi\right] \\
& -i(\omega-n \Omega) \mathbf{Q}=\nabla \times(\mathbf{v} \times \mathbf{B})+(\mathbf{Q} \cdot \nabla \Omega) R^{2} \nabla \phi \\
& \mathbf{p}=p \hat{\mathbf{b}} \hat{\mathbf{b}}+p_{\perp}(\mathbf{I}-\hat{\mathbf{b}} \hat{\mathbf{b}}) \\
& \text { and } p_{\perp}=\sum_{j} \int M_{j} v_{\|}^{2} f_{j}^{1} d \Gamma, \quad p_{\perp}=\sum_{j} \int \frac{1}{2} M_{j} v_{\perp}^{2} f_{j}^{1} d \Gamma
\end{aligned}
$$

where $s$ is the normalized radial coordinate labelling the equilibrium flux surface. More specifically, $s$ is the square root of the normalized equilibrium poloidal flux. $\chi$ is a generalized poloidal angle. $\omega=\omega_{r}+i \gamma$ is the complex eigenvalue of the mode $\left(\gamma\right.$ being the mode growth rate, $\omega_{\mathrm{r}}$ the mode rotation frequency in the laboratory frame). The mode frequency is corrected by a Doppler shift in $\Omega$, with $n$ being the toroidal mode number, $\Omega$ the plasma rotation frequency in the torodial direction $\phi$. A radially uniform flow model is adopted in the present study._ $\xi, \mathbf{v}, \mathbf{Q}, \mathbf{j}, \mathbf{p}$ represent the perturbed quantities: the plasma displacement, the perturbed velocity, magnetic field, current and kinetic pressure tensor, respectively. $\rho$ is the unperturbed plasma density. $\mathbf{B}$ is the equilibrium magnetic field. $R$ is the plasma major radius. $\hat{\mathbf{Z}}$ is the unit vector in the vertical direction. A conventional unit system is assumed with the vacuum permeability $\mu_{0}=1$; and the subsonic plasma flow is assumed. 
The perturbed kinetic pressure tensor $\mathbf{p}$ includes both parallel (to the equilibrium magnetic field), $p_{\|}$, and perpendicular, $p \perp$, components. Each component involves both adiabatic (superscript "a") and non-adiabatic (superscript "na") parts: $p_{\|}=p_{\|}{ }^{a}+p_{\|}{ }^{n a}, p \perp=p \perp^{a}+p \perp^{n a}$.

$p_{g}^{a}=\sum_{j} \int d \Gamma E_{g}\left(-\xi_{\perp} \cdot \nabla f_{j}^{o}\right)$

$p_{g}^{n a}=\sum_{j} d \Gamma E_{g} f_{j}^{1} \quad g=\|, \perp$,

where $E_{\|}=M v_{\|}{ }^{2}$, and $E_{\perp}=M v_{\perp}^{2} / 2=\mu B$ ( $\mu$ is the magnetic moment). $\mathbf{I}$ is the unit tensor, and $\mathbf{b}=\mathbf{B} /|\mathrm{B}| . \Gamma$ denotes the velocity space of particles, and $j$ denotes the particle species including thermal ions and electrons as well as energetic ions $(\mathrm{j}=\mathrm{i}, \mathrm{e}, \mathrm{a}) . f_{\mathrm{j}}^{\mathrm{j}}$ is the equilibrium distribution function. $f_{j}^{l}$ is the solution of the perturbed drift kinetic equation, which we solve together with the fluid equations[10]. Therefore this self-consistent approach provides a drift kinetic closure to the single fluid MHD equations. Besides, a set of vacuum equations for the perturbed magnetic field $\boldsymbol{Q}$, and the resistive wall equation based on the thin-shell approximation, are all solved together with equations (1)-(4) [10].

\subsection{Equilibrium distribution models for particle species}

We assume the Maxwellian equilibrium distribution for thermal ions and electrons. In order to describe the equilibrium distribution of NBI induced EIs, we adopt a model which is slowing down in the particle energy space $\varepsilon_{k}$, and (generally) anisotropic in the particle pitch angle space $\zeta[27,22]$

$f_{a}^{0}\left(\psi, \varepsilon_{k}, \zeta\right)=\left\{\begin{array}{ll}\frac{C(\psi)}{\varepsilon_{k}^{3 / 2}+\varepsilon_{c}^{3 / 2}} \frac{G_{1}}{2 \sqrt{\pi} \delta \zeta}, & \varepsilon_{k}<\varepsilon_{a} \\ 0, & \varepsilon_{k}>\varepsilon_{a}\end{array}\right.$,

where $\varepsilon_{c}=\left(\frac{3 \sqrt{\pi}}{4}\right)^{2 / 3}\left(\frac{M_{a}}{M_{i}}\right)\left(\frac{M_{i}}{M_{e}}\right)^{1 / 3} T_{e}$, with $M_{i}, M_{e}$ and $M_{a}$ denoting the mass of thermal ion, electron and energetic ion, respectively; $\varepsilon_{a}$ is the EIs birth energy. A sum of Gaussian functions, $G_{l}$, is introduced to model the anisotropy in the particle pitch angle $\zeta=v_{\|} / v$

$G_{1}\left(\psi, \varepsilon_{k}, \zeta\right)=\sum_{i=1}^{4} C_{i} \exp \left[-\frac{\left(\zeta-\zeta_{i}\right)^{2}}{\delta \zeta^{2}}\right]$ 
The choices of coefficients $C_{i}$, $\zeta_{i}$ determine various NBI schemes (normal versus tangential) [22]. The width of the Gaussian, $\delta \zeta$, is also a function of the particle energy [22]. Formally as $\delta \zeta$ approaches infinity, the $G_{l}$ factor approaches $2 \sqrt{\pi} \delta \zeta$, and Eq. (8) recovers the isotropic distribution limit for EIs. The factor $C(\psi)$ in Eq. (8) is determined by the EIs (flux surface averaged) density $N_{a}$.

We point out that, generally speaking, the equilibrium distribution of particles is assumed to be a function of three adiabatic invariants: the toroidal canonical angular momentum $P_{\phi}=-Z e \psi+M R^{2}(d \phi / d t)$, the particle energy $\varepsilon_{k}$, and the particle magnetic moment $\left.\mu=M v_{\perp}^{2} / 2 B\right)\left(v_{\perp}\right.$ here is the particle guiding center velocity perpendicular to the equilibrium magnetic field, and B the amplitude of the equilibrium magnetic field strength).In this work, we shall neglect the finite orbit width effect of energetic particles. In this approximation, the second term of the canonical momentum can be dropped. We mention that a formulation with first order finite orbit width correction has also recently been developed [22], that includes the second term in the canonical momentum. The conservation of the particle energy and the magnetic moment implies that the particle distribution is always symmetric with respect to the sign of $v_{\|}$. In reality, transport modelling for EPs often finds asymmetry in the particle distribution along $v_{\|}$. This asymmetry can be due to strange particle orbits [28], and/or asymmetry in the particle losses along the co- and counter-current directions. This asymmetry is captured either by introducing the pitch angle $\zeta$ as in Eq. (8) following Ref. [29], or by explicitly introducing the sign of $v_{\|}, \sigma=\operatorname{sign}\left(v_{\|}\right)$, as the fourth variable in the particle distribution function, as in Ref. [29]. Our model here follows the former approach.

The drift kinetic effects from each species of particles are self-consistently coupled to MHD equations; the detail description can be found in Ref. [10]. For thermal particles, the key element in this formulation is the wave-particle resonance operator, expressed as

$$
\lambda_{m l}^{\alpha}=\frac{n\left[\omega_{*_{N}}+\left(\varepsilon_{k}-3 / 2\right) \omega_{*_{T}}+\Omega\right]-\omega}{n \omega_{d}+[\alpha(m+n q)+l] \omega_{b}+n \Omega-\omega-i v_{e f f}}
$$

where $\omega_{d}$ is the bounce-orbit-averaged precession drift frequency. $\omega_{*_{N}}$ and $\omega_{* T}$ represent the diamagnetic flow frequencies due to the radial gradient of the particle density and temperature, respectively. For trapped particles, $\alpha=0$, and $\omega_{b}$ is the bounce frequency. $l$ is a integer number. For passing particles, $\alpha= \pm l$, and $\omega_{b}$ represents the transit frequency. $v_{\text {eff }}$ is the effective collision frequency of particle species. Similar to thermal particles, the precessional drift resonance operators for fast ions can be expressed as 
$\lambda^{a}=\frac{n\left(\frac{\partial f_{a}^{o}}{\partial \psi} / Z e \frac{\partial f_{a}^{o}}{\partial \varepsilon}\right)-\omega}{n \omega_{d a}+n \Omega-\omega-i \nu_{e f f}}$

where $\omega_{d a}$ is the bounce orbit averaged precessional drift frequency of fast ions. In this work, we shall neglect the finite banana orbit effect.

\section{Kinetic effects on RWMs in RFP plasmas}

\subsection{Specification of toroidal RFP equilibrium}

A toroidal RFP equilibrium is self-consistently computed using the CHEASE code [30], based on the typical plasma parameters from the RFX-mod experiments. The plasma has a circular poloidal cross section, with the inverse aspect ratio of $a / R_{0}=0.2295$. A typical toroidal field reversal factor, of $F=B_{\phi}(a) /\left\langle B_{\phi}\right\rangle=-0.06$, is chosen, with $\left\langle B_{\phi}\right\rangle$ being the volume averaged toroidal field strength. A resistive wall, of circular shape, is radially located at $b / a=1.12$. The plasma current profile is specified such that the on-axis safety factor, $q_{0}$, is 0.1448. The edge safety factor, $q_{a}$, is around -0.09 . Figure 1 shows the radial profile of the safety factor, as well as the locations of various rational surfaces. The plot is extended to the surrounding vacuum region outside the plasma. The RFP configuration is characterised by the reversal of the toroidal magnetic field direction near the plasma edge. RFP can operate in the parameter region where the resonant kink modes (with rational surfaces located inside the plasma) are stable, whilst the non-resonant external kink modes remain unstable without a close-fitting surrounding ideal wall._The poloidal plasma beta is chosen to be $\beta_{p}=8 \pi\left\langle P>V_{\text {tot }} t\left(I^{2} R_{0}\right)=0.155\right.$, where $\langle P\rangle$ is the volume averaged equilibrium plasma pressure $P$, with the total plasma volume of $2 \pi \mathrm{V}_{\text {tot. }} I$ is the plasma current, and $R_{0}$ the major radius of the plasma magnetic axis. The equilibrium electron density is specified by a parabolic radial profile $n_{e}(s)=n_{e}\left(1-s^{2}\right)$, with the plasma core density of $n_{e 0}=2.5 \times 10^{19} / \mathrm{m}^{3}$. 


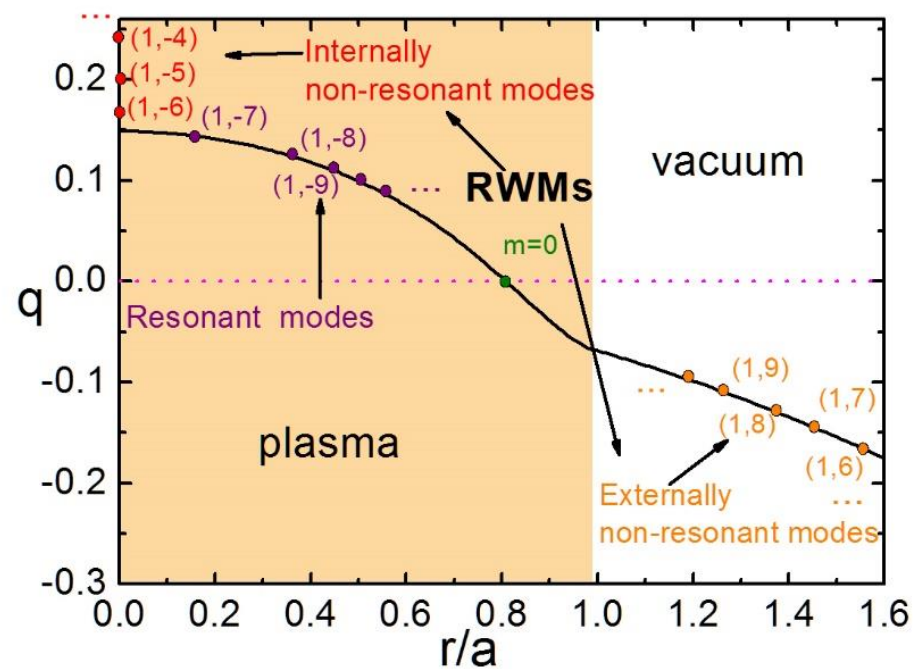

Fig. 1. Radial profile of the safety factor $q$. The RWMs in the RFP plasma have rational surfaces located outside the plasma, and can be classified in two groups: the internal non-resonant mode and the external non-resonant mode. The modes with rational surfaces inside plasma are classified as resonant modes, observed as resistive modes in RFP plasmas.

As descripted in Fig. 1, there are normally two types of RWMs in an RFP plasma: the so called external non-resonant mode (ENRM), with the mode rational surface located at $q<q_{a}<0$ (marked in the vaccum region of the figure); and the internal non-resonant mode (INRM), with the mode rational surface located at $q>q_{0}>0$ (marked at the magnetic axis). Note that both types of mode correspond to the external kink instability, since the mode rational surface is located outside the plasma in both cases. These external kink modes are normally current driven instability in the RFP.

With the aforementioned equilibrium, the most unstable RWM, which we shall study in this work, is the $n=6$ INRM, with the dominant poloidal harmonic number of $m=1$. The corresponding mode rational surface is located at $q=1 / 6>q_{0}=0.1448$. For this $n=6 \mathrm{RWM}$, the ideal wall beta limit, assuming the same ideal wall radial location of $b / a=1.12$ as the resistive wall, is computed to be $\beta_{p}{ }^{i d e a l}=0.16$. The equilibrium that we choose thus is close, but below the ideal wall beta limit - the plasma is in the RWM regime. Under these conditions, the kinetic effects play significant roles in stabilizing the $n=6 \mathrm{RWM}$. 
In this work, we shall specify the equilibrium radial profile for EIs by specifying a ratio $P_{a} / P_{t h}=\beta_{p}{ }^{a} / \beta_{p}{ }^{\text {th }}$, which is generally a function of the plasma minor radius. We also specify the birth energy $\varepsilon_{a}$ of EIs. For a given equilibrium distribution in the particle velocity space, specification of both $\beta^{*}$ and $\varepsilon_{a}$ uniquely defines the density of EIs. The typical values that we choose are $\beta^{*}=P_{a}(0) / P_{t h}(0)=0.3$ and $\varepsilon_{a}=100 \mathrm{keV}$, although these parameters are also varied in the present study. It is important to mention that the total equilibrium pressure is kept the same, while we vary the EIs pressure fraction in this work. This is to ensure that the total MHD drive (including effectively the sum of the adiabatic contributions from all particle species) remains the same, while we vary the relative portion of the (non-adiabatic) kinetic contributions from thermal particles and EIs. An alternative approach, which we do not follow in this work, is to fix the thermal particle pressure while adding/varying the EIs contribution. This will lead to the variation of the total equilibrium pressure, thus changing the MHD drive for the mode.

In the particle velocity space, we assume slowing down distribution in particle energy, and either isotropic or anisotropic distribution in particle pitch angle, as specified in Section 2. In the following, we first report the MARS-K computational results assuming isotropic distribution for EIs.

\subsection{Kinetic effects of EPs with isotropic distribution}

Figure 2 shows a typical example of the kinetic damping results on the $n=6$ RWM in the RFP plasma, including kinetic contributions from either thermal particles or EIs alone, or from both. We consider plasma toroidal flow in both directions. With the adopted sign conventions in MARS-K, the processional drift kinetic resonance occurs when the sum of the precession frequency $\omega_{d}$ of particles and the plasma rotation frequency $\Omega$ vanishes, see Eq. (10). For RFP plasmas, the toroidal precession of particles typically does not experience the so called drift reversal effect in the velocity space. Thus the precession frequency has either positive or negative sign. In fact the precession frequency is always positive (co-current) for trapped ions, and negative (counter-current) for trapped electrons, as shown in Fig. 3. This creates asymmetry effect, with respect to the plasma flow direction, in the kinetic damping due to the precessional drift resonances, in particular for EIs as shown by Figs. 2(a) and (b). In the presence of EIs contribution alone, only the 
counter-current flow, at sufficiently fast speed, leads to the RWM stabilization. The critical plasma rotation frequency, computed for this case, is $\Omega_{C} / \omega_{A}=-0.039$. This frequency also quantitatively matches well the averaged precession frequency of EIs, shown in Fig. 3. No stabilization of the mode is achieved with co-current flow.

The situation is different for thermal particles. First of all, because of the opposite directions of the toroidal precession for thermal ions and electrons, there is always one particle species which is in resonance with the mode, at a given direction of the plasma flow. Therefore, the precessional drift resonance is always present (and reasonably symmetric if $P_{i} \sim P_{e}$ ), independent of the flow direction, as long as the contributions from both thermal ions and electrons are included, which is indeed the case in the present study.

However, for RFP plasmas, the precessional drift resonances do not contribute a major kinetic damping to the RWM, as has been previously reported [15-16] and also confirmed here by Fig. 2. The precessional drift resonances, which occur generally at the plasma rotation frequency $\left|\Omega / \omega_{A}\right|<0.01$, do not stabilize the mode. On the contrary, the full stabilization is achieved at much higher flow speeds (about $-0.039 \omega_{A}$ at counter-current flow and $0.022 \omega_{A}$ at co-current flow), as shown by Fig. 2 . This is primarily due to the transit motion induced kinetic resonances of passing thermal particles [15]. In fact the MARS-K computed critical rotation speeds, shown in Fig. 2, fall well into the transit frequency range for thermal ions, shown in Fig. 3, when the factor $(m-n q+l) / n$ is taken into account in the transit frequency. Note that Fig. 3 only shows the case for $m=1(l=0)$, which gives the primary resonance contribution due to passing particles, for both co- and counter-current flows. The asymmetry in the computed critical rotation speeds, between these two cases, is largely associated with the asymmetry in the nominator of the resonance operator (10). 

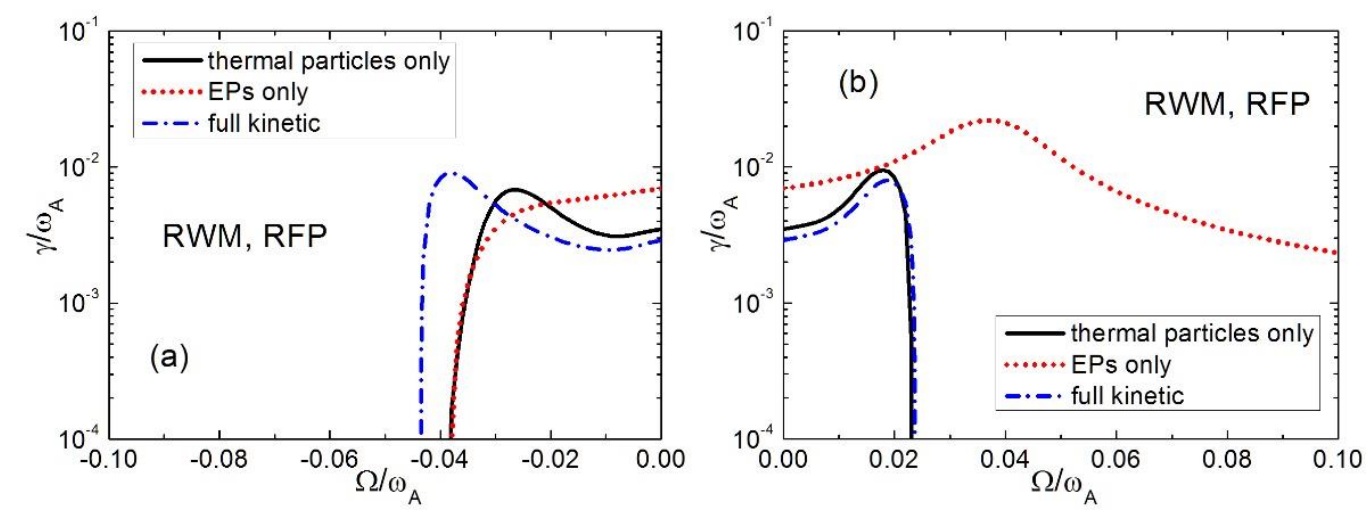

Figure 2. The normalized growth rate $\gamma / \omega_{A}$ of the $n=6 R W M$ versus the toroidal plasma flow in (a) counter-current, and (b) co-current, directions, for a toroidal RFP equilibrium with $\beta_{P}=0.155$, $\beta^{*}=\beta_{h} / \beta_{t h}=$ const $=0.3, F=-0.06, q_{0}=0.145, b / a=1.12$, and $\varepsilon_{\alpha}=100 \mathrm{keV}$. The toroidal angular frequency of the plasma flow, $\Omega / \omega_{A}$, is normalized by the toroidal Alfven frequency on the magnetic axis. Comparison is made between thermal particle kinetic effects alone (solid lines), EPs effects alone (dashed), and the combined effects from both thermal and energetic particles (dash-dotted).

What is more interesting from this study, is the cancellation of the drift kinetic damping for the RWM in RFPs, between the thermal particle and the EIs contributions. As a result, the faster counter-current flow is needed in order to fully stabilize the mode, when all particle contributions are included into the MARS-K computations, as shown in Fig. 2(a). Such a cancellation does not occur for the co-current flow case, since no drift kinetic resonances occur for EIs in this case. Consequently, the predicted critical rotation speeds are nearly the same, with or without the EIs contribution.

We emphasize that two conditions have to be satisfied, in order for the aforementioned cancellation to occur. First, both thermal and EIs, individually, need to be in resonance with the mode. Secondly, and perhaps more importantly, both resonances (with thermal and energetic particles) have to occur at similar toroidal rotation speed of the plasma. In our RFP plasma, the counter-current flow case satisfies both conditions. In particular, the second condition is satisfied between the transit frequency of passing thermal ions and the precession frequency of trapped EIs. 


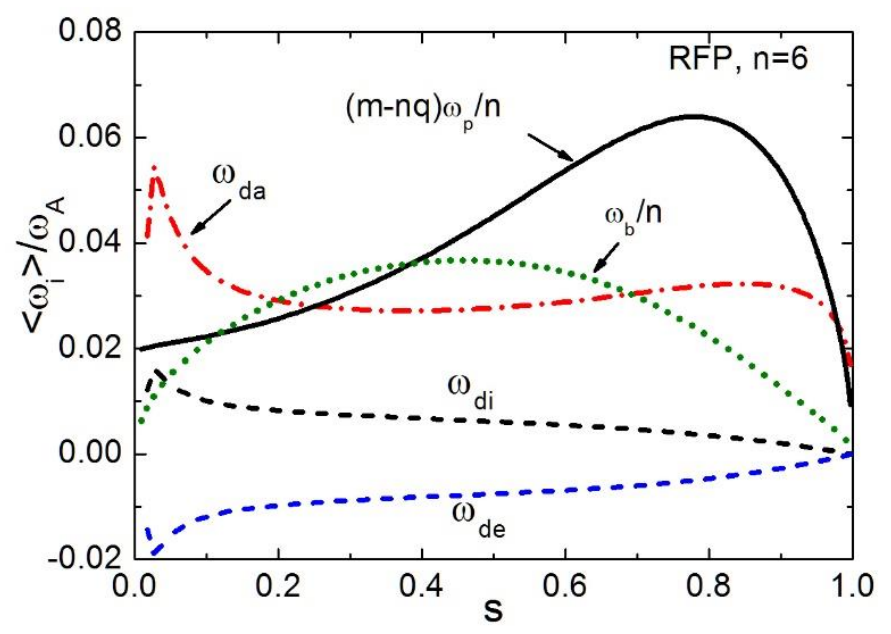

Figure 3. Various drift frequencies of particle species, averaged over the particle velocity space as well as over the poloidal angle, and plotted along the plasma minor radius $s$, including the transit frequency $(m-n q) \omega_{p} / n \quad(m=1)$ of passing thermal ions, the bounce frequency $\omega_{b} / n$ of the trapped thermal ions, the toroidal precession frequencies $\omega_{d i}, \omega_{d e}$ of trapped thermal ions and electrons, respectively, as well as the precession frequency $\omega_{d a}$ of trapped energetic ions. The equilibrium parameters are the same as in Fig.2.

Figure 3 presents the radial profiles of various drift frequencies, averaged over the poloidal angle as well as over the particle velocity space, for the same RFP equilibrium parameters as that in Fig.2. The precession frequency of energetic ions, the transit frequency of the passing thermal ions, and the bounce frequency of the trapped thermal ions, as well as the precession frequencies of thermal trapped ions and electrons are all plotted for comparison. It shows that the transit frequencies and bounce frequency of the thermal ions are located in the frequency range, which is comparable with the precession frequency of EIs. Therefore, at a certain range of the plasma rotation, if the RWM is in resonance with the precession frequency of EIs, it may also be in resonance with the transit and bounce frequencies of thermal ions. Each species can make their contribution, respectively, to the drift kinetic potential energy. The stability nature of the RWM is determined by the combination of the kinetic effects from the two species. The trapped thermal particles have much lower precession frequencies, compared to that of EIs, thus giving a negligible influence on the cancelation effects in the RFP plasmas. 
The direct evidence of the cancellation is shown in Fig. 4, where we compare the imaginary parts of the net perturbed drift kinetic contributions from different particle species, while varying the plasma flow speed in both directions. The imaginary parts of $\delta W_{k}$ represent the drift kinetic resonances, and always play the most important stabilizing role on the RWM, independent of the sign. We point out that the net perturbed kinetic energies are evaluated, based on the self-consistently computed mode eigen-frequency and eigen-function by MARS-K, at each flow speed. Note that the cancellation occurs only for the counter-current flow case. For the co-current flow case, the imaginary part of $\delta W_{k}$ vanishes from the EIs contribution, since no drift kinetic resonance occurs. We also note that the cancellation shown in Fig. 4(a) is generally not complete.
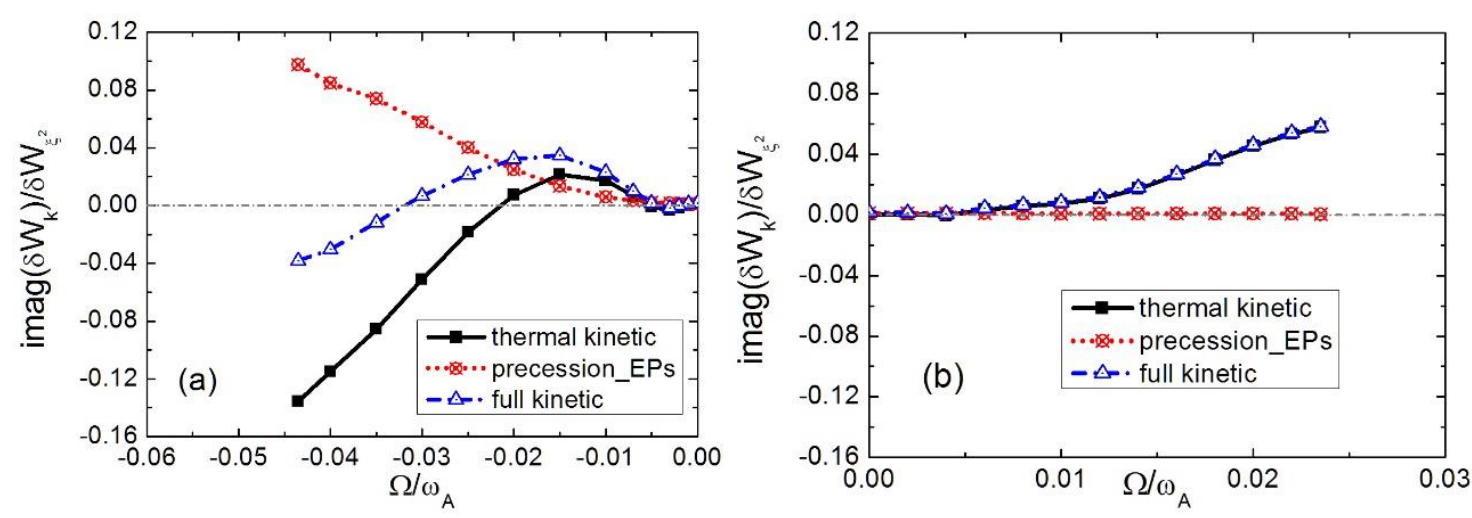

Figure 4. The imaginary parts of the net drift kinetic energy perturbations, normalized by the perturbed inertial energy and plotted as functions of (a) counter-current, and (b) co-current, toroidal rotation frequency. Comparison is made between thermal particle kinetic effects alone (solid lines), EPs effects alone (dashed), and the combined effects from both thermal and energetic particles (dash-dotted). The equilibrium parameters are the same as in Fig.2.

According to the dispersion relation for the RWM, a partial cancellation of the net kinetic energy damping terms (between thermal and energetic ions in our case) leads to reduced stabilization of the mode. On the other hand, the self-consistent computations with MARS-K does not only involve the integrated energies, and we expect that local cancellation of the damping energy also takes place. This is indeed the case. As an example, Fig. 5 shows local quantities, associated with trapped EIs, in real space for (a) the precessional drift frequency averaged over the particle velocity space, and (b) the imaginary part of the perturbed kinetic energy at flow speed $\Omega / \omega_{A}=-0.035$. 
Figure 5(a) shows a large flat region located primarily in the low field side (LFS) of the torus, where the phase space averaged precession frequency of trapped EIs is in the range of $\omega_{d} \sim 0.03-0.04 \omega_{A}$. Therefore, a strong resonance is expected to occur when the mode frequency, which is essentially the Doppler shifted frequency due to the plasma flow in the plasma frame, matches $n \omega_{d}$. Indeed, large amplitude of $\operatorname{Im}\left(\delta W_{k}\right)$ is also observed in the LFS region, at the matching flow speed $\Omega / \omega_{A}=-0.035$, as shown by Fig. 5(b). The energy perturbation is more localized near the plasma core, due to the large mode structure (the plasma displacement) of the dominant $\mathrm{m}=1$ harmonic, which is more localized near the core [16]. We emphasize that the discussion of the frequency matching in Fig. 5 is still approximate, since the real resonance occurs locally also in the particle velocity space (both particle energy and pitch angle).
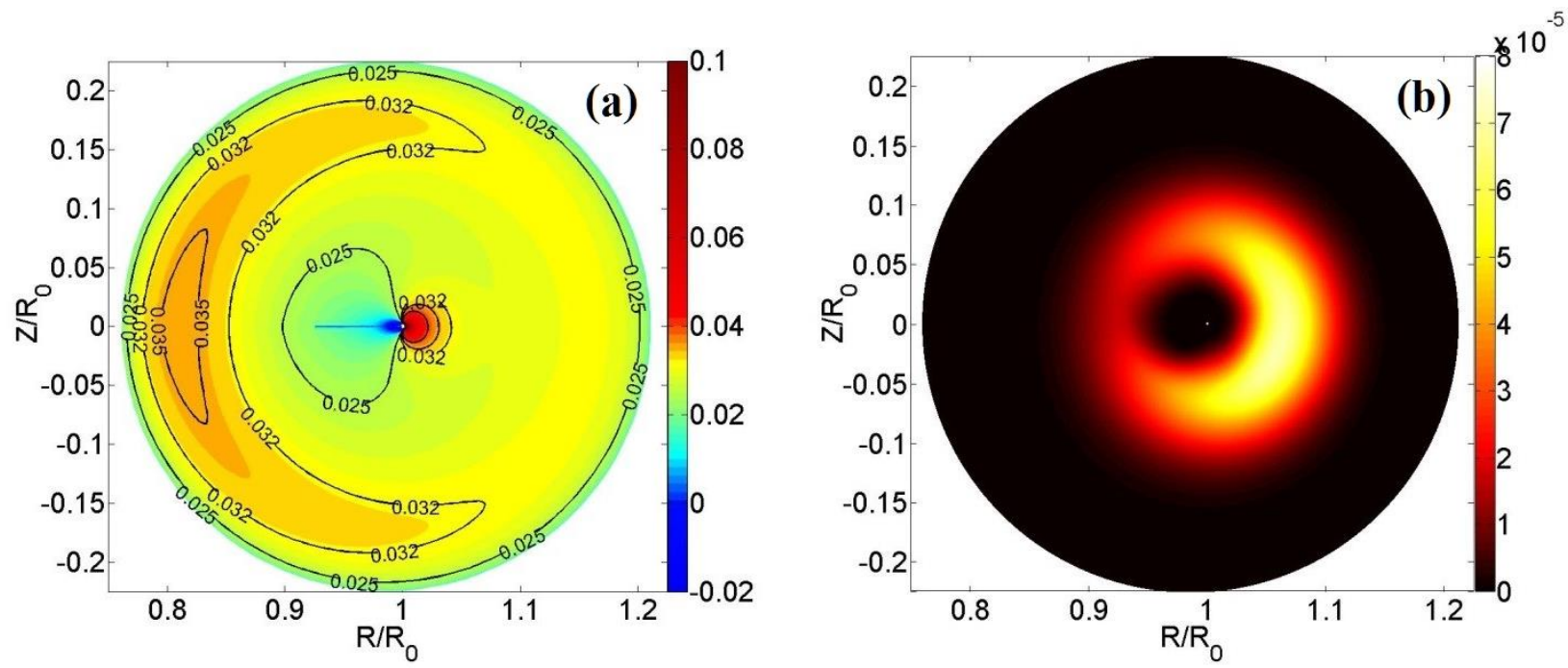

Figure 5. Plotted on the poloidal cross section of the RFP plasma are (a) the precessional drift frequency of trapped energetic ions, averaged over the particle velocity space, and $(b)$ the imaginary part of the perturbed drift kinetic energy from trapped EIs alone, assuming a toroidal rotation frequency of $\Omega / \omega_{A}=-0.035$. The other equilibrium parameters are the same as that in Fig. 2.

We also find a similar local distribution of $\operatorname{Im}\left(\delta W_{k}\right)$ in the R-Z plane but with the opposite sign, from the transit resonance contribution of passing thermal ions, thus confirming the local cancellation of the perturbed drift kinetic energy in real space, between the thermal and energetic ions. 
We point out that the $\operatorname{Im}\left(\delta W_{k}\right)$-based interpretation (Figs.4-5) of the RWM stabilization/destabilization (Fig. 2) is strictly valid only for the perturbative approach for the RWM stability analysis. In the self-consistent approach, which is adopted in this study, $\operatorname{Im}\left(\delta W_{k}\right)$ itself is a (non-linear) function of the mode's eigenvalue. Therefore, the stabilizing role of $\operatorname{Im}\left(\delta W_{k}\right)$ is more difficult to analyze. However, the a-posteriori analysis as we performed here in Figs. 4-5 (as well as in later figures for the tokamak equilibrium), where $\operatorname{Im}\left(\delta W_{k}\right)$ is evaluated by inserting the eventual mode's eigen-frequency as obtained from the self-consistent computations, still follows the similar physics in terms of the stabilizing role played by $\operatorname{Im}\left(\delta W_{k}\right)$.

One further question to ask is how the eventual stabilization of the RWM is still possible, when the cancellation of the drift kinetic damping energy occurs. Figure 4 offers a partial answer - the amount of the net damping energy, after cancellation, increases with faster plasma flow. More understanding is gained by varying the birth energy of EIs, thus varying the precession frequency of EIs as shown in Fig. 6(b). The precession frequency of the trapped EIs is proportional to the particle birth energy $\varepsilon_{a}$, as expected. By fixing the EIs density fraction, we also vary the EIs pressure, which linearly scales with $\varepsilon_{a}$. Since the total equilibrium pressure is kept unchanged (satisfying the equilibrium force balance), the thermal particle pressure (and hence the thermal particle temperature) also varies with $\varepsilon_{a}$, resulting in the pressure fraction parameter $\beta^{*}$ varying faster than $\varepsilon_{a}$. Therefore, with increasing $\varepsilon_{a}$, the precession frequency of trapped EIs (linearly) increases, whilst the bounce or transit frequency of thermal particles decreases (as square root of the particle energy). This changes the resonance conditions for both thermal and energetic particles, at a given plasma flow speed, thus gives us another tool to investigate the cancellation effect.
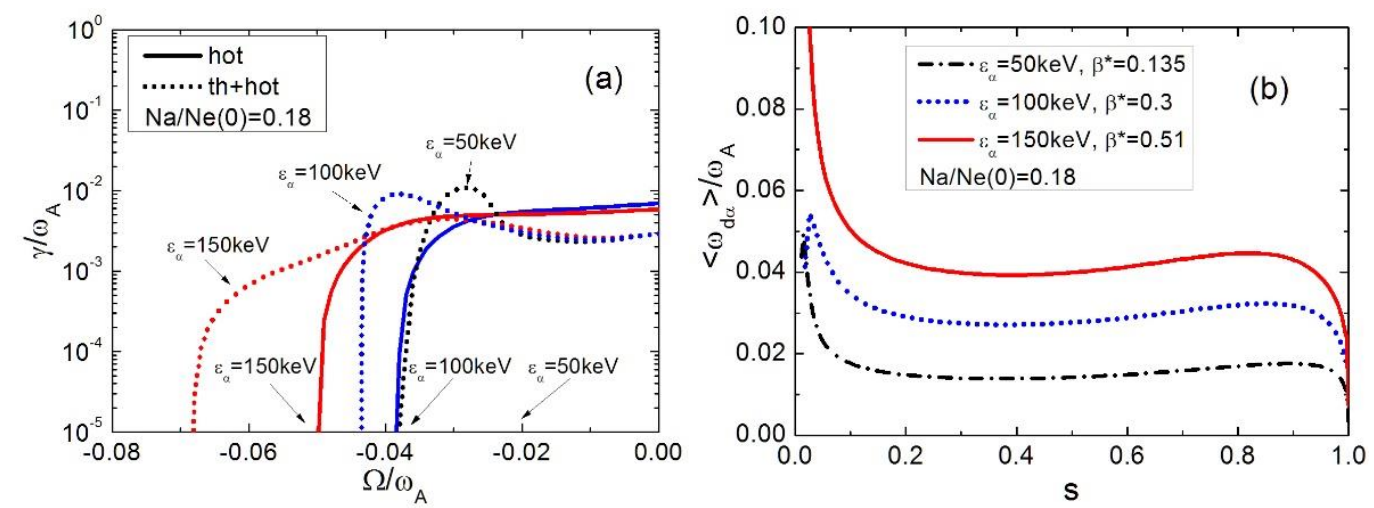
Figure 6. Comparison of (a) the growth rate of the $n=6$ RWM versus the counter-current plasma flow frequency, and $(b)$ the toroidal precession frequency of EIs, averaged over the particle velocity space as well as over the poloidal angle, and plotted versus the plasma minor radius, with three choices of the EIs birth energy $\varepsilon_{\alpha}=50 \mathrm{keV}\left(\beta^{*}=0.135\right), 100 \mathrm{keV} \quad\left(\beta^{*}=0.3\right), 150 \mathrm{keV} \quad\left(\beta^{*}=0.51\right)$. The growth rates with the EIs kinetic contribution alone (solid lines), and with both EIs and thermal particle contributions (dashed), are also compared in (a). Note that the total equilibrium plasma pressure, as well as the EIs density fraction, is kept unchanged while varying the EIs birth energy. The other equilibrium parameters are the same as that in Fig. 2.

The final results are summarized in Fig. 6(a). At low birth energy $\left(\varepsilon_{a}=50 \mathrm{keV}\right)$, the precession frequency of EIs is also low $\left(\omega_{d a} \sim 0.02 \omega_{A}\right)$. The $n=6 \mathrm{RWM}$ is fully stabilized at low plasma rotation $\Omega=-0.021 \omega_{A}$, with the contribution from EIs alone. Inclusion of the thermal particle contribution, at this flow frequency, leads to large cancellation, and hence the loss of the RWM stabilization. However, with further increasing the flow speed, the resonance with EIs diminishes. At $\Omega \sim-0.039 \omega_{A}$, the kinetic damping from thermal ions dominates, and the mode is again stabilized. No significant cancellation occurs at this flow speed.

On the other hand, the high birth energy $\left(\varepsilon_{a}=150 \mathrm{keV}\right)$ of EIs increases the precession frequency up to $\omega_{d a} \sim 0.05 \omega_{A}$. The mode is fully stabilized at plasma rotation of $\Omega=-0.05 \omega_{A}$, with the contribution from EIs alone. Inclusion of thermal particle contribution again leads to the cancellation and thus the loss of the RWM stability at this flow speed. The cancellation is stronger because of the larger fraction of EIs pressure in this case. As a result, even faster plasma flow is required for the stabilization of the mode, when both thermal and hot ions contributions are included.

\subsection{Kinetic effects of EPs with anisotropic distributions}

In this work, the anisotropy of the EIs equilibrium distribution, in the particle pitch $\zeta$, is modelled by Gaussian functions $G_{l}$ as defined by Eq. (9). In the following, we choose the coefficients $C_{i}$ and $\zeta_{i}$ such that both normal and co-current tangential NBI injections are modelled. Examples of the $G_{l}$ factor, as functions 
of $\zeta$, are shown in Fig. 7(a), for the isotropic distribution, for the co-tangential NBI induced EIs, as well as for two normal NBI injection cases with different Gaussian width $\delta \zeta_{0}\left(\delta \zeta_{0}\right.$ is formally the value of $\delta \zeta$ at infinite particle energy). In our study, only trapped EIs are considered. These are particles with pitch angle $|\zeta|<0.4$ in our examples. With the same number of total EIs, tangential injection gives the least fraction of trapped particles, as expected. Normal injections give larger fraction of trapped EIs, than the isotropic model. On the other hand, the normal injection model, with a larger Gaussian width (dash-two dotes line in Fig. 7(a)), gives less trapped hot ions than the normal injection with a smaller Gaussian width (dash dote line in Fig.7), results in a distribution that is close to isotropic.

Figure 7(b) compares the MARS-K computed growth rates for the $n=6 \mathrm{RWM}$, assuming various EIs distributions as reported in Fig. 7(a). The drift kinetic contributions from thermal particles are always present in all cases. The results again clearly illustrate the cancellation effect as we already find for the isotropic distribution case, and their consequence on the mode stability.

The most stable case, in terms of the required minimal critical flow speed for full stabilization of the mode, is obtained with the co-tangential injection model. This is because this case suffers the least cancellation effect, due to the small fraction of trapped EIs. In such a case, the dominant contribution for the mode damping comes from the thermal ions. The isotropic model results in a larger fraction of trapped EIs, compared to the co-tangential case. As a result, larger degree of cancellation occurs, and a faster flow is needed to fully stabilize the mode.
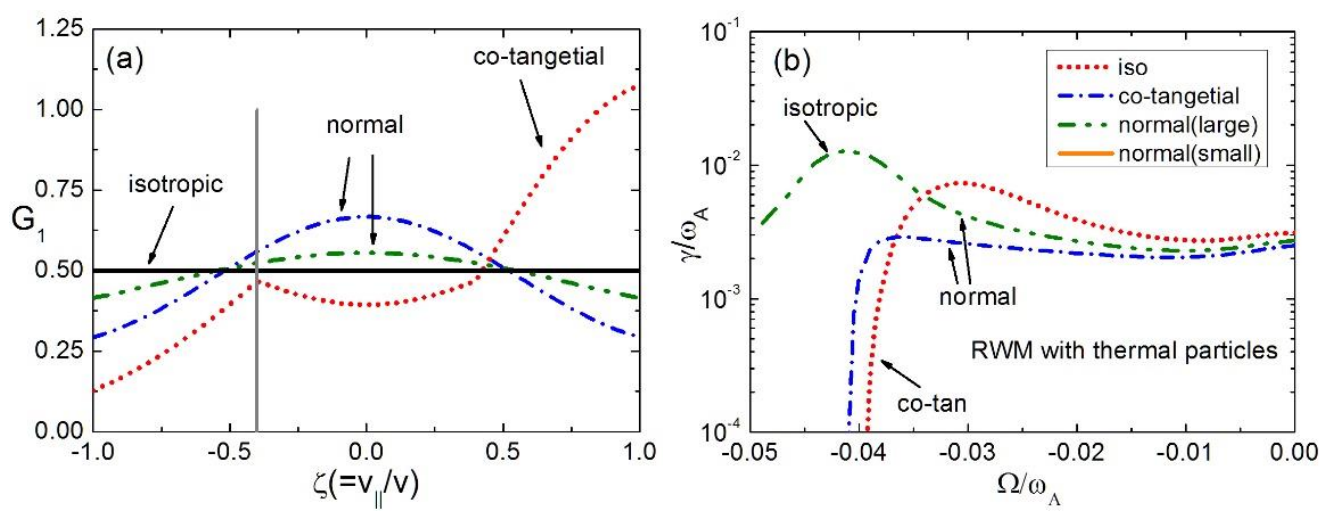
Figure 7. The effects of (a) the anisotropy (specified by the $G_{1}$ factor as defined in the text) of the EIs equilibrium distribution in the particle pitch angle space $\zeta$, on (b) the stability of the RWM, when the drift kinetic effects from both EIs and thermal particles are taken into account. Considered is the counter-current flow case, with all other equilibrium parameters chosen the same as that in Fig. 2. The two vertical lines in (a) indicate the trap-passing boundary.

At the other extreme, where the fraction of trapped EIs is the largest (normal injection with small Gaussian width $\left(\delta \zeta_{0}=0.8\right)$ ), a relatively small critical rotation is again observed for the mode stabilization. This is because the EIs kinetic contribution now over-compensates that of thermal ions. Therefore, in this case, the dominant role of the kinetic damping for the $n=6$ RWM is played by EIs.

Perhaps the most interesting case is the normal injection with large Gaussian width $\left(\delta \zeta_{0}=1.2\right)$. Here the trapped fraction of EIs is such that neither thermal nor energetic ions kinetic contribution is dominant, thus a good cancellation occurs for a wide range of the plasma flow. As a result, the mode stays unstable even at fast plasma flow.

\section{Kinetic effects on RWMs in tokamak plasmas}

\subsection{Specification of toroidal plasma equilibrium}

We wish to understand whether the aforementioned cancellation effect, observed for the $n=6 \mathrm{RWM}$ in the RFP plasma, can also occur for low- $n$ RWM in a tokamak plasma. For the purpose of a comparative study, we choose a tokamak equilibrium with the same plasma shape (circular) and the same inverse aspect ratio $(a / R=0.2295)$, as that for the RFX-mod plasma. The on-axis vacuum toroidal and the resistive wall minor radius are also the same, with $B_{T}=1.5 \mathrm{~T}$ and $b / a=1.12$, respectively. A major difference with the RFP equilibrium is obviously the safety factor $q$. We choose a plasma current profile such that the on-axis $q$-value is $q_{0}=1.1$, and the edge value is $q_{a}=3.6$. The corresponding radial $q$ profile is plotted in Fig. 8 , where the $q$-profile is extended to the surrounding vacuum region outside the plasma. The equilibrium toroidal beta value is chosen to be $\beta=0.011$ (the normalized beta is $\beta_{\mathrm{N}}=3.0$ ) for this tokamak plasma. This pressure level 
is close to the ideal-wall beta limit, of $\beta=0.012\left(\beta_{\mathrm{N}}=3.2\right)$, for the onset of the $n=1$ external kink instability in this plasma. This is the regime where the drift kinetics have strong effect on the stability of the RWM, essentially due to the fact that the perturbed fluid potential energy $\delta W_{b}$, with the ideal wall, approaches zero and is thus easily perturbed by the kinetic energy $\delta W_{k}[15]$.

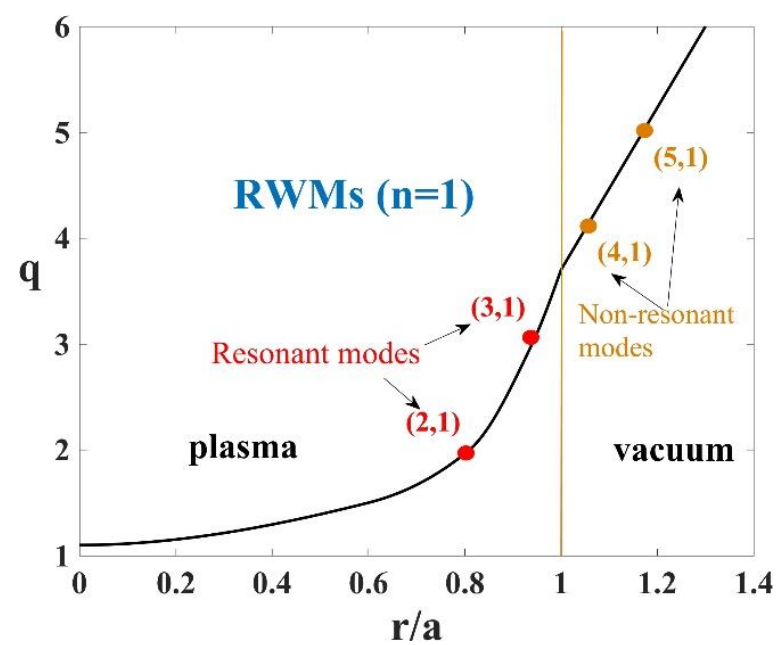

Fig. 8. Radial profile of the safety factor $q$ in the tokamak plasma, with $q(0)=1.1$ and $q(a)=3.6$. The locations of rational surfaces correspond to the resonant $(m=2,3)$ and non-resonant $(m=1,4,5)$ harmonics of the $n=1$ mode.

In what follows, we shall study the stability of the $n=1$ RWM for this tokamak equilibrium. Similar to the study for the RFP plasma, we shall include the drift kinetic resonances associated with: toroidal precession of thermal ions, electrons and energetic ions; the bounce and transit motions of thermal ions. The other drift motions of particles (e.g. bounce/transit motions of thermal electrons or fast ions) are neglected for the RWM study, since these are normally too fast (due to either too light particle mass or too large particle kinetic energy) to generate kinetic resonances with the mode. We shall only assume the isotropic EIs model in the tokamak study, which is sufficient to illustrate the cancellation physics.

\subsection{Kinetic effects of EPs with isotropic distribution}

The drift kinetic damping of the RWM by thermal particles, in a tokamak plasma, is somewhat different from that in an RFP, as has previously been shown [16]. In the tokamak case, the primary damping comes 
from the precessional drift resonances of trapped thermal ions and electrons, whilst in an RFP, the primary damping of the mode comes from passing thermal ions. This is also confirmed by comparing Figs. 7(a) and 1(a) in this study.

For the tokamak case shown in Fig. 9, in the absence of the EIs contribution, the $n=1$ RWM is fully stabilized at very slow toroidal flow speed of the plasma, in the frequency range of $\Omega / \omega_{A} \sim[-0.003,0.004]$. This rotation frequency range, which also corresponds to the real mode frequency in the rotating plasma frame, largely matches that of the precessional drift frequency of thermal particles shown in Fig. 10, but is far below the bounce/transit frequencies of thermal ions. Therefore, even though all the aforementioned kinetic effects are included into the MARS-K computations for this case, the resonance damping due to the precessional drifts of thermal particles is the key factor leading to the mode stabilization at slow flow. The nearly symmetric effect of the mode damping, with respect to the counter- or co-current flow, is due to the fact that both thermal ion and electron contributions are included. Therefore, at a given sign of the flow speed, one of the particle species will be in resonance, producing a similar amount of the damping energy. At faster flow, resonances with the bounce/transit motions of thermal ions become more prominent. But still, no full stabilization of the mode is achieved with the thermal particles alone.
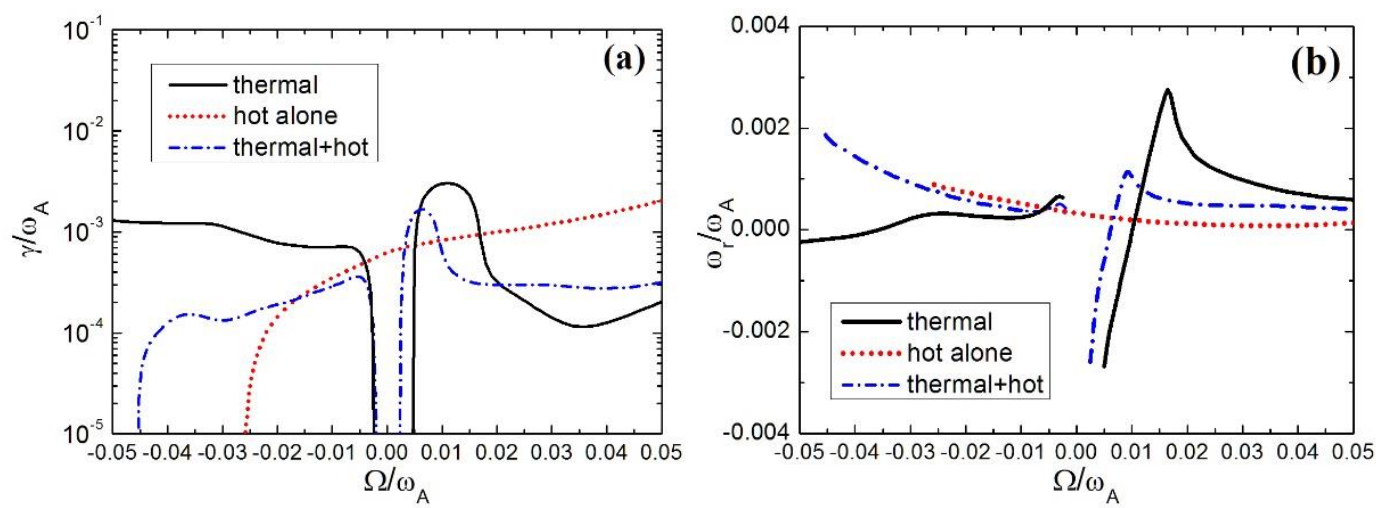

Figure 9. The (a) growth rate $\gamma / \omega_{A}$, and (b) real frequency, of the $n=1 R W M$ versus the toroidal plasma rotation frequency $\Omega / \omega_{A}$ in both directions, for a tokamak plasma equilibrium. Compared are cases with thermal particle kinetic contribution alone (solid lines), with EIs alone (dashed), and with both thermal particle and EIs contributions combined (dash-dotted). The EIs pressure fraction, with birth energy of 
$\varepsilon_{\alpha}=100 \mathrm{keV}$, is specified as $\beta_{h} / \beta_{t h}=\beta^{*}\left(1-s^{2}\right)^{8}$, with $\beta^{*}=0.19$. The other equilibrium parameters are $\beta=0.011, \quad \beta_{N}=3.0$ and $b / a=1.12$.

On the other hand, inclusion of the EIs contribution alone breaks the aforementioned symmetry for thermal particles. In fact stabilization of the mode is achieved only with the counter-current flow. This is qualitatively similar to the RFP case shown in Fig. 2(a), with a similar physics reason as discussed before. The critical flow speed, for the complete mode stabilization, is $\Omega / \omega_{A} \sim-0.026$ for this case.

Combining together the kinetic contributions from both EIs and thermal particles, we observe two interesting effects. First, EIs marginally affect the precessional drift kinetic damping of the mode due to thermal particles, at slow plasma flow. (In fact a slight narrowing of the stability window is obtained at co-current flow, by adding the EIs contribution. But the effect is small.) This is because EIs precessional motion does not produce significant resonances with the mode at this slow flow (though a moderate amount of resonances are indeed created as will be shown later). Secondly and more interestingly, adding the kinetic contribution of thermal particles (primarily thermal ions), at fast flow, reduces the damping effect produced by the trapped EIs. This occurs with both flow directions, although the full stabilization is achieved again only with counter-current flow. The critical rotation speed is significantly increased by the addition of the thermal particle contribution. This is again due to the cancellation of the drift kinetic damping energy between thermal and energetic ions, similar to what has been observed (Fig. 2) for the RFP plasma.

Finally, we note that the real frequency of the mode, computed in the wall frame and shown in Fig. 9(b), is generally small and stays within the range for a typical RWM. In other words, we are dealing with the RWM branch, even in the presence of EIs. 
Figure 10 presents radial profiles of various drift frequencies, averaged over the poloidal angle as well as over the particle velocity space, for the same equilibrium parameters for tokamak as that in Fig.9 . It shows the similar conclusion as shown in Fig. 3 for RFP plasmas, namely, at a certain range of the plasma rotation, if the RWM is in resonance with the precession frequency of EIs, it may be in resonance with the transit frequency of passing thermal ions and bounce frequencies of trapped thermal ions as well. Furthermore, in tokamaks, the precession frequency of EIs in certain area is much lower (near zero) than that in RFP plasmas. Therefore, even in the slow rotation regime, the precession motion of trapped EIs can still be in resonance with the mode, contributing non-negligible drift kinetic energy.

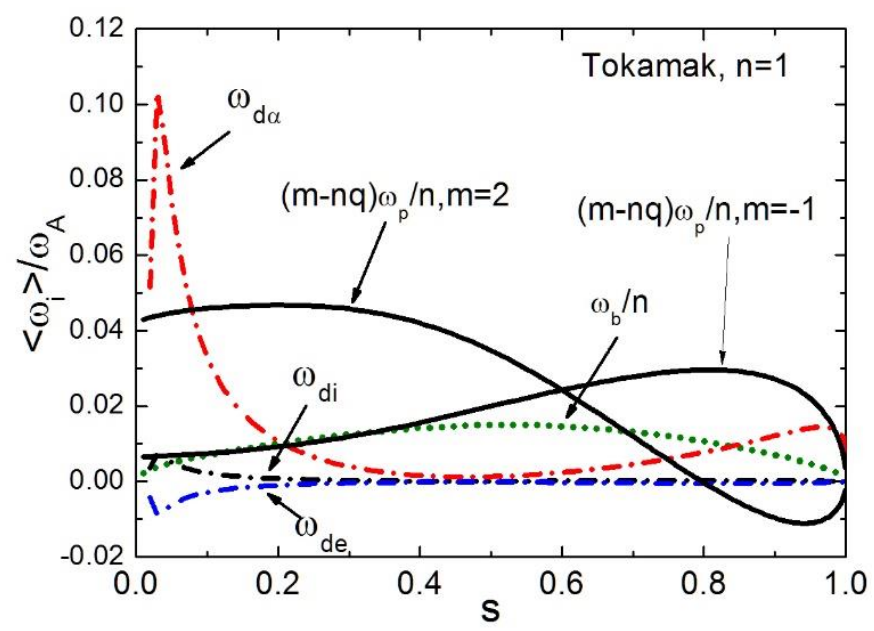

Figure 10. The radial profiles of various particle drift frequencies, averaged over the particle velocity space and over the poloidal angle, in a tokamak equilibrium for the toroidal precession frequency $\omega_{d i}, \omega_{d e}$ of trapped thermal ions and electrons, respectively; the precession frequency $\omega_{d \alpha}$ of trapped EIs with the birth energy of $\varepsilon_{\alpha}=100 \mathrm{keV}$; the bounce frequency $\omega_{b} /$ nof trapped thermal ions; as well as the transit frequencies $(m-n q) \omega_{p} / n$ of passing thermal particles, associated with different poloidal harmonics that participate into the mode-particle resonance condition. The equilibrium parameters are chosen the same as in Fig. 9.

Figure 11 provides a more detailed analysis of the perturbed energy distributions among various particle species and different types of kinetic resonances. The imaginary parts of each energy components are compared, for three choices of the plasma rotation frequencies: $\Omega / \omega_{A}=-0.043, \Omega / \omega_{A}=-0.003$, and 
$\Omega / \omega_{A}=+0.003$. At fast counter-current flow $\Omega / \omega_{A}=-0.043$, both transit resonance of passing thermal ions and precession resonance of trapped EIs contribute, and partially cancel each other. The dominant contribution for the mode damping comes from EIs. At slow counter-current flow $\Omega / \omega_{A}=-0.003$, the dominant contribution is due to the precession resonance of trapped thermal ions. The EIs also contribute a small amount which leads to the enhancement of the damping. The other contributions are negligible. At slow co-current flow $\Omega / \omega_{A}=+0.003$, the largest contribution to the mode damping comes from the precession resonance of trapped thermal electrons. The other contributions, being small, partly cancel the electron contribution. In particular, the EIs contribution partly cancels the electron contribution, leading to the slight destabilization of the mode at slow co-current flow, as shown in Fig. 9. This energy balance analysis thus fully supports the computed mode stability shown in Fig. 9.

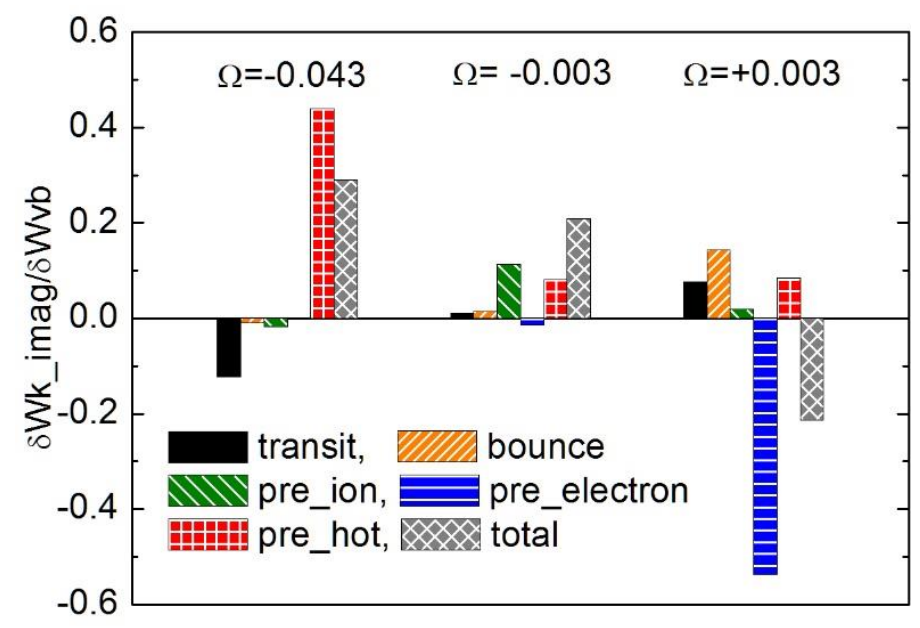

Figure 11. Comparison of various contributions to the imaginary part of the perturbed drift kinetic energy, from transit resonance of passing thermal ions (transit), bounce resonance of trapped thermal ions (bounce), precessional drift resonance of trapped thermal ions (pre_ion), precessional resonance of trapped thermal electrons (pre_electron), and precessional resonance of EIs (pre_hot). Three plasma rotation speeds are assumed, with $\Omega / \omega_{A}=-0.043, \Omega / \omega_{A}=-0.003$, and $\Omega / \omega_{A}=+0.003$, respectively. The other equilibrium parameters are the same as that in Fig. 9.

The energy histogram from Fig. 11 also shows one interesting feature, i.e. a small but finite amount of perturbed kinetic energy from EIs produced at slow flow, independent of the flow direction. This is indeed possible by analyzing the local frequency of toroidal precession of trapped EIs in real space, as shown in Fig. 12(a). Similar to Fig. 5(a) for the RFP equilibrium, this frequency is obtained after the average over the particle velocity space. However, different from the RFP case, there is a considerable region in the R-Z 
plane shown in Fig. 12(a), where the drift frequency is in the counter-current direction (with negative values). This eventually results in resonance between EIs and the mode at co-current flow.
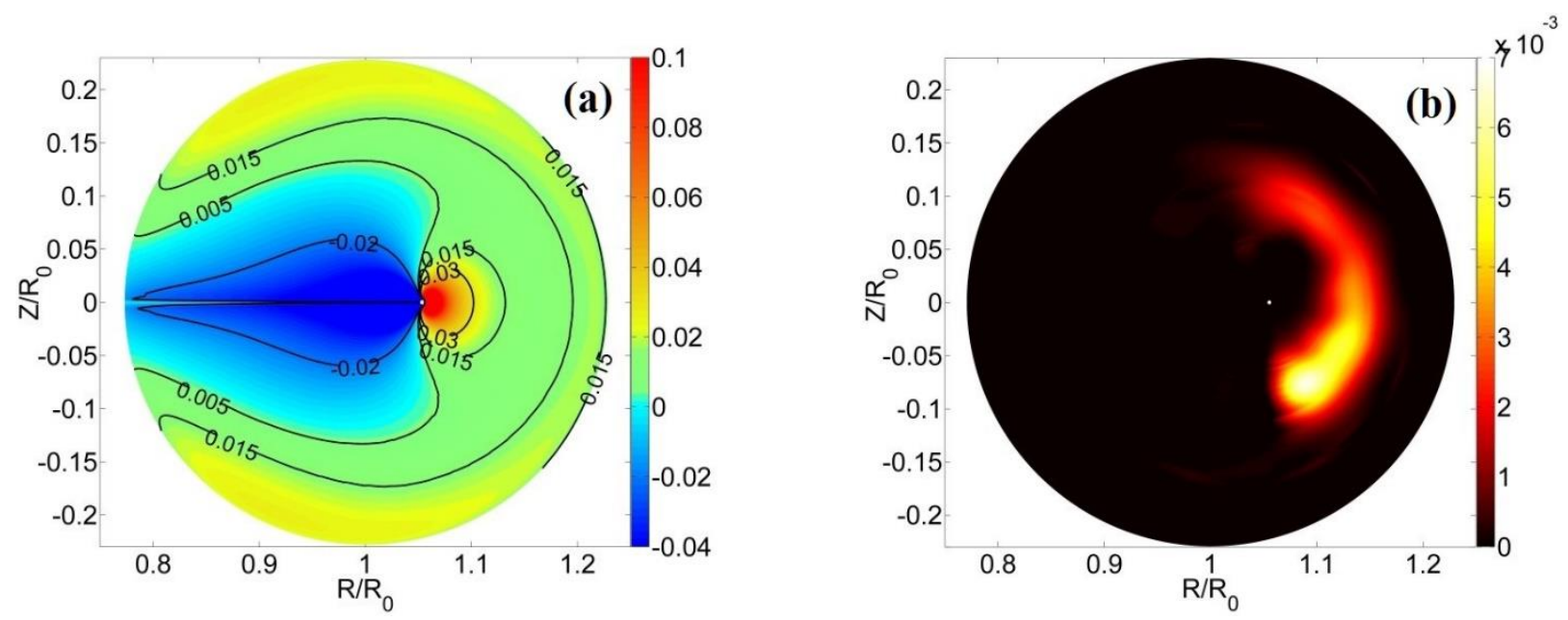

Figure 12. Plotted on the poloidal cross section of the tokamak plasma are (a) the precessional drift frequency of trapped energetic ions, averaged over the particle velocity space, and $(b)$ the imaginary part of the perturbed drift kinetic energy from trapped EIs alone, assuming a toroidal rotation frequency of $\Omega / \omega_{A}=-0.02$. The other equilibrium parameters are the same as that in Fig. 9..

Furthermore, Fig. 12(a) shows a large region of co-current toroidal precession, at averaged frequencies of $\sim 0.02 \omega_{A}$. The mode-particle resonance in this frequency range leads to the stabilization of the RWM by EIs alone, as shown in Fig. 9(a). The 2D distribution of the MARS-K computed damping energy from EIs, for the flow speed of $\Omega / \omega_{A}=-0.02$, is plotted in Fig. 12(b). Similar to the RFP plasma, significant amount of the damping energy, for this $n=1$ RWM, is located in the LFS of the torus. The up-down asymmetry of the energy distribution is attributed to the plasma flow effect.

\section{Summary and conclusion}

In this work, we have performed computational study of the drift kinetic effects of both thermal and energetic particles on the stability of the RWMs, in both toroidal RFP and tokamak plasmas, using the well benchmarked non-perturbative MHD-kinetic hybrid code MARS-K. The key result is a finding of cancellation effect of the kinetic damping on the RWM, between the thermal particles and EIs contributions, 
in both RFP and tokamak configurations. In terms of the energy perturbation, the cancellation results in the reduction of the imaginary part of the total perturbed drift kinetic energy.

The drift kinetic resonances, that we include into the non-perturbative computations, include the precessional drift of trapped thermal ions and electrons, of Maxwellian equilibrium distribution; the bounce and transit drift motions of trapped and passing thermal ions, respectively; the precessional drift of trapped EIs, of either isotropic or anisotropic equilibrium distribution in the particle pitch angle space and slowing down distribution in particle energy. The EIs are assumed to possess finite birth energies, of typical values for neutral beam induced particles in present day toroidal fusion devices.

For the RFP plasmas, either thermal particles or EIs alone can stabilize the most unstable RWM (with $n=6$ in our chosen toroidal equilibrium). Whilst the thermal particle stabilization, primarily resulting from the transit resonance of passing thermal ions, occurs for co- and counter-current toroidal flows, the precessional drift resonance stabilization from EIs occurs only for the counter-current flow, at which the proper resonance conditions can be satisfied. The cancellation between the thermal and energetic ions kinetic effects thus takes place with the counter-current plasma flow.

A comparative study has also been carried out for the $n=1 \mathrm{RWM}$, based on a toroidal tokamak plasma with the same plasma shape (circular poloidal cross section), inverse aspect ratio $(a / R=0.2295)$, toroidal equilibrium field (1.5T), and the wall minor radius $(b / a=1.12)$. The EIs kinetic effects on the $n=1 \mathrm{RWM}$ is qualitatively similar in the tokamak plasma, i.e. full stabilization of the mode at fast counter-current toroidal plasma flow, compared to that on the $n=6$ RWM in the RFP. However, the effects of thermal particles are different than that in RFP, on two major aspects: (i) full stabilization of the $n=1$ RWM is achieved at very slow plasma flow, primarily due to the precessional drift resonance of trapped thermal ions and electrons; (ii) no full stabilization of the mode is achieved at fast flow, despite the inclusion of bounce and transit resonances of thermal particles. Despite this difference, a similar cancellation effect is obtained for the 
tomaka plasma, where adding the thermal particle contribution reduces the kinetic damping effect introduced by the EIs.

On the other hand, since the cancellation effect studied in this work is sensitive not only to the plasma flow, but also to the equilibrium distribution of EIs. It is possible to minimize the cancellation of the damping energy on the RWM, by tailoring the distribution of trapped EIs.

As a final remark, we mention that this partial cancellation effect between thermal and energetic particles not only occurs for the damping of the RWM, but also during the triggering of the fishbone-like external kink modes (FLEM) by EIs in tokamaks and RFPs, as has recently been predicted by the MARS-K computations [31]. The difference is that the same (cancellation) effect leads to the reduced damping (and hence destabilization) of the RWM, whilst to the reduced efficiency of triggering (and hence stabilization) of the FLEM.

\section{Acknowledgements}

The authors acknowledge very helpful discussions with Dr. Deyong Liu on the energetic particle equilibrium distributions in RFP plasmas.

This work has been carried out within the framework of the EUROfusion Consortium and has received funding from the Euratom research and training programme 2014-2018 under grant agreement No 633053 and from the RCUK Energy Programme [grant number EP/I501045]. Work is also part funded by National Natural Science Foundation of China (NSFC) [grant numbers 11428512] and by National Magnetic Confinement Fusion Science Program under grant No. 2015GB104004. The views and opinions expressed herein do not necessarily reflect those of the European Commission.

\section{References}

[1] Bondeson A. and Ward D.J. 1994 Phys. Rev. Lett. 722709

[2] Hender T.C. et al Progress in the ITER Physics Basis Chapter 3: MHD stability, operational limits and disruptions 2007 Nucl. Fusion 47 S128-S202

[3] Chu M.S. and Okabayashi M. 2010 Plasma Phys. Controlled Fusion 52123001 
[4] Liu Y.Q. and Bondeson A. 2000 Phys. Rev. Lett. 84907

[5] Bondeson A. et al 2001 Nucl. Fusion 41455

[6] Liu Y.Q. et al 2010 Plasma Phys. Controlled Fusion 52104002

[7] Baruzzo M. et al 2012 Nucl. Fusion 52103001

[8] Martin P. et al 2009 Nucl. Fusion 49104019

[9] Bo H. and Betti R. 2004 Phys. Rev. Lett. 93105002

[10] Liu Y.Q. et al 2008 Phys. Plasmas 15112503

[11] Liu Y.Q. et al 2008 Phys. Plasmas 15092505

[12] Chapman I.T. et al 2009 Plasma Phys. Controlled Fusion 51055015

[13] Zheng L.J. et al 2010 Phys. Plasmas 17056104

[14] Berkery J.W. et al 2010 Phys. Plasmas 17082504

[15] Wang Z.R. et al 2012 Nucl. Fusion 52063001

[16] Wang Z.R. et al 2012 Phys. Plasmas 19072518

[17] Guo S.C. et al 2013 Nucl. Fusion 53113035

[18] Liu Y.Q. et al 2009 Nucl. Fusion 49035004

[19] Xia G.L. et al 2015 Nucl. Fusion 55093007

[20] Liu Y.Q. 2010 Nucl. Fusion 50095008

[21] Hao G.Z. et al 2011 Phys. Rev. Lett. 107015001

[22] Liu Y.Q. et al 2014 Phys. Plasmas 21056105

[23] He Y.L. et al 2014 Phys. Rev. Lett. 113175001

[24] Chapman I.T. et al 2012 Phys. Plasmas 19052502

[25] Sarff J.S. et al 2015 Nucl. Fusion 55104006

[26] Guo S.C. et al 1999 Phys. Plasmas 63868

[27] Gorelenkov N.N. et al 2005 Nucl. Fusion 45226 
[28] White R.B., The theory of toroidally confined plasmas (Chapter 3), Second edition, 2001, Imperial College Press.

[29] Porcelli F. et al 1994 Phys. Plasmas 1470

[30] Lutjens H. et al 1996 Comput. Phys. Commun. 97219

[31] Guo S.C. et al 2016 Nucl. Fusion 56056006 